\title{
On neutrino-atom scattering in searches for neutrino magnetic moments
}

\author{
Konstantin A. Kouzakova ${ }^{\mathrm{a}}$, Alexander I. Studenikin ${ }^{\mathrm{b}, \mathrm{c}, *}$, Mikhail B. Voloshin ${ }^{\mathrm{d}, \mathrm{e}}$ \\ ${ }^{a}$ Department of Nuclear Physics and Quantum Theory of Collisions, Faculty of Physics, Moscow State University, Moscow 119991, Russia \\ ${ }^{b}$ Department of Theoretical Physics, Faculty of Physics, Moscow State University, Moscow 119991, Russia \\ ${ }^{c}$ Joint Institute for Nuclear Research, Dubna 141980, Moscow Region, Russia \\ ${ }^{d}$ William I. Fine Theoretical Physics Institute, University of Minnesota, Minneapolis, Minnesota 55455, USA \\ ${ }^{e}$ Institute of Theoretical and Experimental Physics, Moscow, 117218, Russia
}

\begin{abstract}
In the experimental searches for neutrino magnetic moments using germanium detectors one studies the ionization channel in the neutrino-atom scattering. We find that the so-called stepping approximation to the neutrino-impact ionization is exact in the semiclassical limit, and that the deviations from this approximation are very small.
\end{abstract}

The neutrino magnetic moments (NMM) expected in the Standard Model are very small and proportional to the neutrino masses: $\mu_{v} \approx 3 \times 10^{-19} \mu_{B}\left(m_{v} / 1 \mathrm{eV}\right)$, with $\mu_{B}=e / 2 m_{e}$ being the electron Bohr magneton, and $m_{e}$ is the electron mass. Any larger value of $\mu_{v}$ can arise only from physics beyond the Standard Model [1]. Current direct experimental searches for a magnetic moment of the electron (anti)neutrinos from reactors have lowered the upper limit on $\mu_{v}$ down to $\mu_{v}<3.2 \times 10^{-11} \mu_{B}$ [2]. At small energy transfer $T$ the inclusive cross section for the magnetic neutrino scattering on a free electron behaves as $d \sigma_{(\mu)} / d T \propto 1 / T$ [3], while that due to weak interaction, $d \sigma_{(w)} / d T$, is practically constant in $T$ [3]. The current experiments using Ge detectors have reached threshold values of $T$ as low as few $\mathrm{keV}$, where one can expect a modification of the free-electron formulas due to the binding of electrons in the Ge atoms. In a recent paper [4], a significant enhancement of the NMM contribution by the atomic ionization effects was claimed. And later on, the authors of Ref. [4] disproved their claim (see also Refs. [5, 6]). Our recent theoretical analysis [7], involving the WKB and Thomas-Fermi models and accounting for electronic correlations, has shown that the

\footnotetext{
${ }^{*}$ Corresponding author

Email address: studenik@srd.sinp.msu.ru (Alexander I. Studenikin)
}

so-called stepping approximation (SA), originally introduced in Ref. [8] from an interpretation of numerical data, works with a very good accuracy. SA treats the process as scattering on independent electrons occupying atomic orbitals and suggests that the cross sections follow the free-electron behaviors down to $T$ equal to the ionization threshold for the orbital; and below that energy the electron on the corresponding orbital is 'inactive' thus producing a sharp 'step' in the dependence of the cross section on $T$. We thus argue that SA can be applied to the analysis of the present and future data of searches for NMM with Ge detectors down to the values of the energy deposition $T \sim 0.3 \mathrm{keV}$.

One of the authors (A.I.S.) thanks Prof. Tzanakos for the invitation to attend the Neutrino 2010 Conference.

\section{References}

[1] C. Giunti and A.I. Studenikin, Phys. At. Nucl. 73 (2009) 2089.

[2] A.G. Beda et al. (GEMMA collaboration), arXiv:0906.1926 [hep-ex]; arXiv:1005.2736 [hep-ex].

[3] P. Vogel, J. Engel, Phys. Rev. D 39 (1989) 3378.

[4] H.T. Wong, H.-B. Li, S.-T. Lin, Phys. Rev. Lett. 105 (2010) 0161801, arXiv:1001.2074 [hep-ph].

[5] M.B. Voloshin, Phys. Rev. Lett. 105 (2010) 201801.

[6] K.A. Kouzakov, A.I. Studenikin, Phys. Lett. B 696 (2011) 252.

[7] K.A. Kouzakov, A.I. Studenikin, M.B. Voloshin, arXiv:1101.4878 [hep-ph].

[8] V.I. Kopeikin, L.A. Mikaelyan, V.V. Sinev, S.A. Fayans, Yad. Fiz. 60 (1997) 2032 [Phys. At. Nucl. 60 (1997) 1859]. 